\title{
Improved Optimal Slotted CSMA/CA Protocol
}

\author{
Nimarta Sidhu \\ Department Of Computer \\ science, \\ GTBKIET,Chhapianwali \\ Malout, Punjab \\ India
}

\author{
Paramjeet Singh \\ Department Of Computer \\ science, GZSCET PTU campus \\ Bathinda, Punjab \\ India
}

\author{
Shaveta Rani \\ Department Of Computer \\ science, GZSCET PTU campus \\ Bathinda, Punjab \\ India
}

\begin{abstract}
This research work focuses on designing an optimal slotted CSMA/CA. The main motivation is the cycles wasted in unslotted and slotted CSMA/CA. So to reduce wasted cycles a new technique is proposed in this research work which use adaptive slots also called optimal slots for Slotted CSMA/CA handles collision by reducing the wasted cycles The proposed protocol is designed and implemented in MATLAB. The simulation has shown significant improvement over slotted and un-slotted CSMA/CA based upon some performance parameters.
\end{abstract}

\section{General Terms}

Mobile Ad hoc network, Optimal Slotted CSMA/CA protocol.

\section{Keywords}

Carrier Sense Multiple Access/Collision Avoidance(CSMA/CA) Request-To-Send (RTS), Clear-ToSend (CTS),Acknowledgement(ACK).

\section{INTRODUCTION}

Carrier sense multiple access/collision avoidance (CSMA/CA) is a multiple access method implemented in wireless networks for sensing the collisions and avoiding them from occurring. For efficient use of wireless channels among multiple nodes, CSMA/CA relies on the random deferment of packets. This class of MAC protocols is one of the most popular for ad hoc networks. A network can be defined as a connection between communicating devices. Everything from Bluetooth device, to Wi-Fi computer, to cell phone operates on a network. Networks come in all sizes, shapes and complexities. Wired networks provides stability and security though they are difficult to setup. Wireless networks are easy to setup but are hard to keep secure. Implementing a secure combination of both wired and wireless network is the most challenging task. Consultant's advice is suggested while making a choice for the network. Selecting the right network is vitally important for getting the most from the infrastructure.

This research work deals with reducing the overheads of slotted CSMA/CA by improving the slotted time of the existing slotted CSMA/CA in such a way that it will provide lower delay and also will not increase the cost of existing slotted CSMA/CA. The Cost and delay depends upon the time slotted CSMA/CA take to do its decision making. The main attention of this research work is to understand the working of slotted CSMA/CA and change time interval (slot) in such a way that it becomes adaptive. A suitable simulation is performed and various parameters regarding wireless networks and CSMA/CA are calculated in order to analyse the performance. (CSMA/CA) access method as the name indicates has several characteristics in common with
CSMA/CD. The difference is in that instead of detecting collisions CSMA/CA attempts to avoid them.

\subsection{Wireless Networking}

Wireless networks are becoming very popular and easy to set up. Although setting up a wireless network is becoming easier, doing it correctly is still challenging. Two major challenges faced in wireless networks are (i) communication over wireless link (ii) mobile users who change point of attachment to network. Wireless networks are now faster, have broader ranges, and are more reliable than they used to be but they are also easy to hack, suffer from various form of interface, and can drop devices from the network if they are not setup properly. It is advisory to take network expert's advice while implementing it.

\subsection{Wired Networking}

Wired networks are the fastest and most reliable networks, but they are difficult to implement. Complicated computer configuration, need of additional hardware and various wiring standards need expert's approach. Frequently many homes and offices are turning to wireless networks, but the wireless networks are not secure enough. Wireless networks face decreased signal strength and interference from various sources. Making communication across wireless link is difficult as compared to wired link. When we require reliability, speed, and security of a wired network it is vital that we consult a professional who can help to plan and implement the network properly.

\subsection{Ad-Hoc Network}

A wireless Ad hoc network is a decentralized type of wireless network. Ad hoc network does not rely on pre existing infrastructure. In this network each node participates in routing by forwarding data to other nodes. Determination of node forwarding data is done dynamically based on connectivity. An ad hoc network refers to any set of networks where all devices have equal status on a network and are free to associate with any other Ad hoc network device in the link range. An ad hoc network, or MANET (Mobile Ad hoc Network), is a network composed only of nodes, with no Access Point. An ad hoc network has the capability of making communications possible even between two nodes that are not in direct range with each other. Packets to be exchanged between two nodes are forwarded by intermediate nodes, using a routing algorithm. Hence, a MANET may spread over a larger distance, provided that its ends are interconnected by a chain of links between nodes. VANET (vehicular Ad hoc network) is a type of mobile Ad hoc network used for communication between vehicles and roadside equipments. iMANET is another type of Ad hoc network that link mobile nodes and fixed internet gateway nodes. 


\subsection{CSMA/CA}

Carrier sense multiple access with collision avoidance (CSMA/CA) has been adopted by the IEEE 802.11 standards for wireless local area networks (WLANs). CSMA/CA sense and avoid collisions, thus improving performance. Distributed coordination function (DCF) of CSMA/CA protocol reduces collisions and improves the overall throughput. To lessen fairness issues arising with slotted CSMA/CA in wireless networks, a modified version CSMA/CCA was proposed which provides copying collision avoidance by copying the size of $\mathrm{CW}$ (contention window).

\section{$1.5 \mathrm{CSMA} / \mathrm{CA}$ working}

On a network that uses the CSMA/CA access method, when a computer has data to transmit, its NIC first checks the cable to determine if there is already data on the wire. So far, the process is identical to CSMA/CD. However, if the NIC senses that the cable is not in use, it still does not send its data packet. Instead, it sends a signal of intent--indicating that it is about to transmit data to the cable, that signal is RTS (request to send). After receiving RTS, the destination waits for a period called Short interframe space (SIFS). If the channel is sensed free the intended node is sent a CTS (Clear to send) signal. The node then transmits the data and the recipient node sends an ACK (acknowledgment) after successful reception of data. Destination sends ACK after waiting for SIFS. CSMA/CA (Carrier Sense Multiple Access/Collision Avoidance) is the channel access mechanism used by most wireless LANs in the ISM bands.

\subsection{CSMA/CA and Wireless LAN}

Wireless LAN cannot implement CSMA/CD for three reasons:

1. Station must be able to send and receive data at the same time.

2. Collision may not be detected because of the hidden terminal problem.

3. Distance between stations in wireless LANs can be larges. Signal fading could prevent a station at one end from hearing a collision at other end.

\section{RELATED WORK}

Zhang, Yijin et al. [1] has proposed and validated an analytical model for the capacity throughput of the IEEE 802.15.4 slotted CSMA/CA which can be studied as slotted non-persistent CSMA. Key to the accuracy of the model is a careful study of the idle period after last collision, which has not been considered by all previous 802.15.4 models.

Pollin, et al. [2] has studied one of the MAC schemes proposed in slotted carrier sense multiple access with collision avoidance (CSMA/CA), and has analyzed whether this scheme meets the design constraints of those low-power and low-cost sensor networks.

Ben othmon jalel et.al [3] has proposed a new MAC protocol that considers different types of traffic and assigns a different priority level to each type of traffic. To improve the Qos of IEEE 802.11 Mac protocol over multichannel CSMA/CA protocol a new admission policy is developed for both voice and data traffic.

Wang, Feng et al. [4] has analyzed the carrier sense multiple access (CSMA) mechanism, and simulated its application in IEEE 802.15.4. Wang, Feng et al. [4] has found that the throughputs are relevant to the normalized propagation delay and the basic time period (BTP) of slotted CSMA. The throughputs of slotted and un-slotted CSMA are almost equal when the normalized propagation delay is much less than 1 and the BTP is equal to the propagation delay.

Wen et al. [5] has proposed IEEE 802.15.4 protocol to meet the low latency and energy consumption needs in low-rate wireless applications. However, few analytical models are tractable enough for comprehensive evaluation of the protocol.

Wang, et al. [6] has shown that when an IEEE 802.15.4 network has many nodes and is almost saturated, the probability of collision is large, and the throughput is small.

Jing, Hui, and Hitoshi Aida (2011) [7] in paper "An analytical approach to optimization of throughput for IEEE 802.15.4 slotted CSMA/CA networks" has discussed the most widely implemented standards for wireless networks, IEEE 802.15.4 defines physical and MAC specifications for low data rate wireless personal area networks (WPANs).

Jing, Hui, and Hitoshi Aida [8] has discussed the most widely implemented standards for wireless networks IEEE 802.15.4 which defines physical and MAC specifications for low data rate wireless personal area networks (WPANs).

Alvi [9] has discussed the IEEE 802.15.4 standard specifically designed for low Rate Wireless Personal Area Network (LR-WPAN) with low data rate and low power capabilities. Due to very low power consumption with duty cycle even less than 0.1 , the standard is being widely applied in Wireless Sensor Networks applications.

\section{PROPOSED PROTOCOL}

Fig 1 shows the flowchart of the proposed protocol. A node which want to send data, start sending, if the channel is sensed free. If the medium is busy, the node continues to monitor until the channel is idle. Then it starts sending data. If the channel is sensed free, the node starts sending the packet. Otherwise, the node waits for a random amount of time (slot) and then monitors the channel, and sends data after the slotted time interval. In improved slotted CSMA/CA, if the channel gets free at run time the node is free to transmit the data. Working of protocol in steps is as follow

Step 1: Wireless network initialization.

Step 2: Define parameters:

First define number of stations

Define radio range of stations

Set time slot of random motion.

Set Average transmission time.

Step 3: Network is initialized and it is assumed that user has started the transmission. Firstly the collision will be checked, if no collision is detected the process of transmission will start.

Step 4: If collision is detected, then wait for slotted time and try again after slotted time.

Step 5: If the channel gets idle before the slotted time ( at run time) transmit the data 


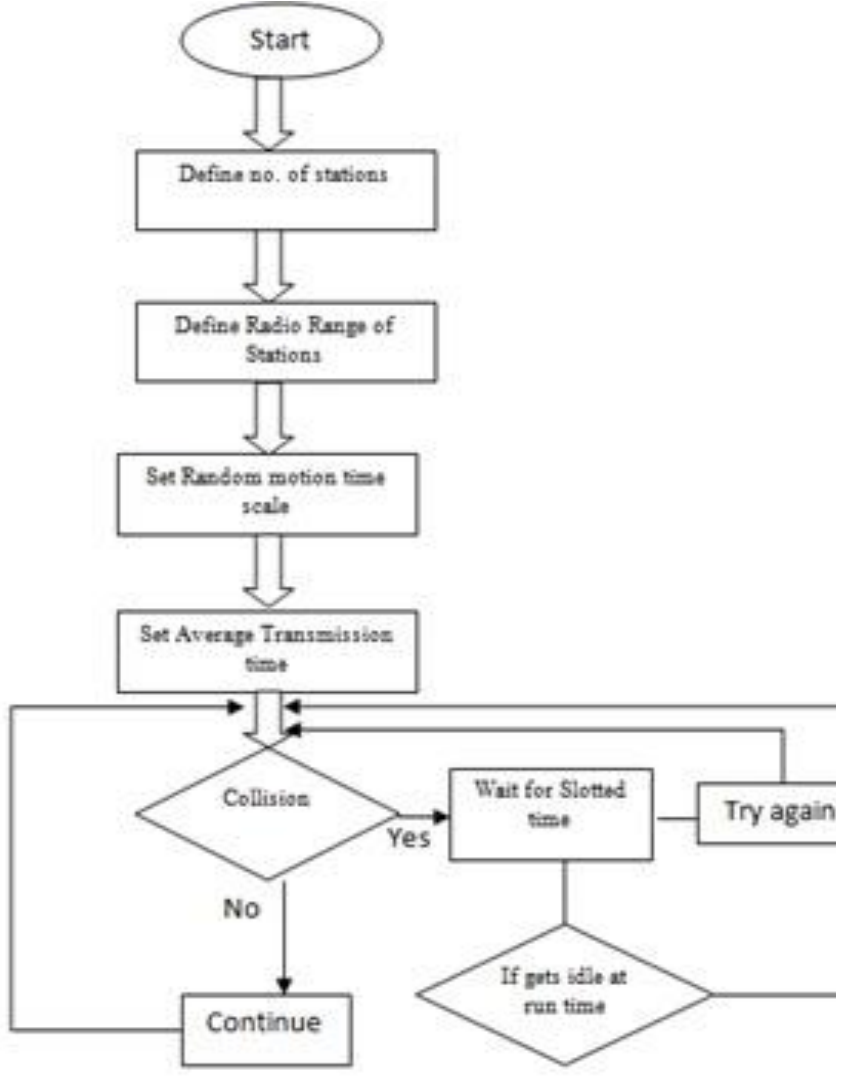

Fig 1 Flow chart of Improved optimal slotted CSMA/CA

\section{EXPERIMENT RESULTS}

Due to the non-availability of the physical environment suitable simulation will be done in the MATLAB. The proposed algorithm is designed and run in the MATLAB by considering various scenarios of CSMA/CA protocol. A script is developed in MATLAB which behave same as slotted CSMA/CA protocol. To do performance analysis multiple simulation results of proposed technique is compared with existing unslotted and slotted CSMA/CA.

Fig 2 shows the partial results of simulation, which is clearly showing the data packets inside transmission range with cyan circle and the packets outside the transmission range with magenta circle. Red line shows the user's request for transmission and the green line indicates acknowledgment.

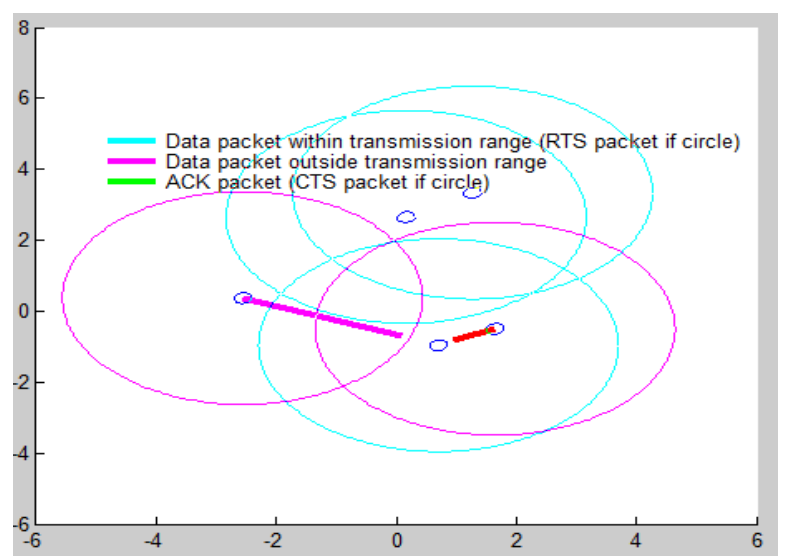

Fig 2 Results of simulation
Fig 3 is showing the partial results of simulation, which is clearly defining the data packets which are inside and outside the transmission range and two red lines from different source nodes are indicating collisions.

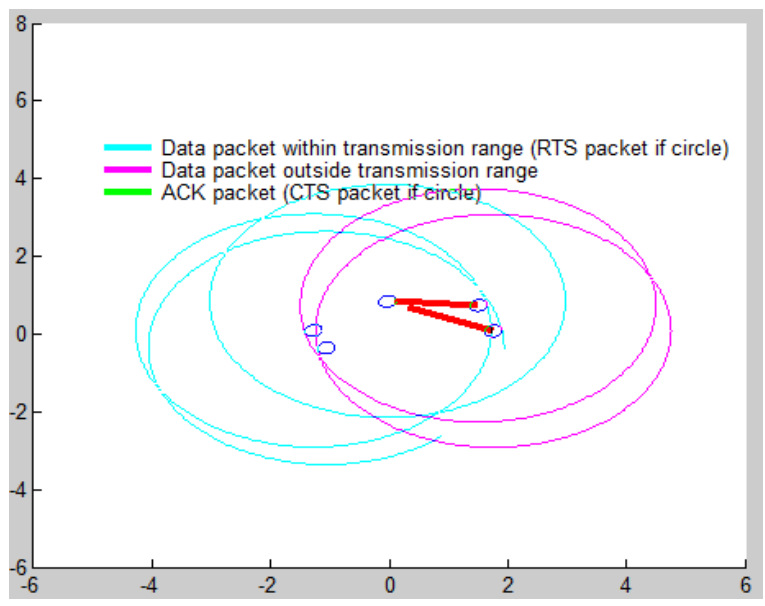

Fig 3 Results of simulation

\section{PERFORMANCE ANALYSIS}

By implementing the slotted CSMA/CA, un-slotted CSMA/CA and proposed algorithm in MATLAB we have taken the following results. Fig 4 shows the parameters for analysing the performance of improved optimal slotted, Slotted and unslotted CSMA/CA. Table 1 is showing the throughput comparison of the slotted CSMA/CA, un-slotted CSMA/CA and the proposed algorithm. Table 2 indicates the delay due to spinlocking and Table 3 shows the delay due to early request finish. It is observed that improved optimal slotted CSMA/CA gives far better results as compared to the existing CSMA/CA techniques.

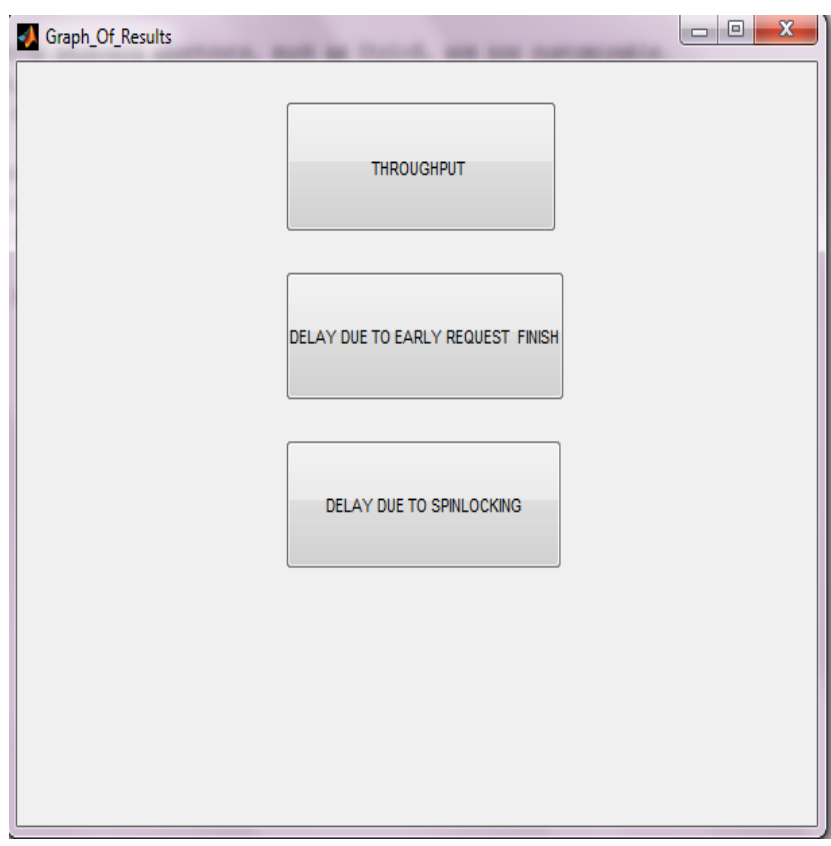

Fig 4 Parameters for performance analysis 
Table 1 Throughput Analysis

\begin{tabular}{|l|l|l|l|}
\hline $\begin{array}{l}\text { No. of } \\
\text { tries }\end{array}$ & $\begin{array}{l}\text { Optimal } \\
\text { slots }\end{array}$ & Slotted & Un-slotted \\
\hline 300 & 200 & 175 & 165 \\
\hline 320 & 214 & 189 & 177 \\
\hline 340 & 227 & 199 & 179 \\
\hline 360 & 240 & 212 & 197 \\
\hline 380 & 254 & 222 & 218 \\
\hline 400 & 267 & 231 & 221 \\
\hline 420 & 280 & 245 & 227 \\
\hline 440 & 294 & 258 & 240 \\
\hline 460 & 307 & 266 & 249 \\
\hline 480 & 320 & 283 & 263 \\
\hline 500 & 334 & 288 & 266 \\
\hline 520 & 347 & 307 & 286 \\
\hline 540 & 360 & 316 & 307 \\
\hline 560 & 374 & 330 & 306 \\
\hline 580 & 387 & 341 & 301 \\
\hline 600 & 400 & 351 & 321 \\
\hline & & & \\
\hline
\end{tabular}

Table 1shows that the throughput of improved optimal slotted CSMA/CA at multiple no of tries is better than unslotted and slotted CSMA/CA.

Table 2 Delay due to spin locking

\begin{tabular}{|l|l|l|l|}
\hline $\begin{array}{l}\text { No. of } \\
\text { tries }\end{array}$ & $\begin{array}{l}\text { Optimal } \\
\text { slots }\end{array}$ & Slotted & $\begin{array}{l}\text { Un- } \\
\text { slotted }\end{array}$ \\
\hline 30 & 189.60 & 240 & 291.60 \\
\hline 320 & 186 & 254.40 & 310.80 \\
\hline 340 & 210 & 271.20 & 351.60 \\
\hline 360 & 213.60 & 288 & 360 \\
\hline 380 & 224.40 & 302.4 & 357.60 \\
\hline 40 & 252 & 319.20 & 390 \\
\hline 420 & 254.40 & 336 & 428.40 \\
\hline 440 & 271.20 & 350.40 & 470.40 \\
\hline 460 & 273.60 & 367.20 & 460.80 \\
\hline 480 & 284.40 & 384 & 469.20 \\
\hline 50 & 297.60 & 398.40 & 501.60 \\
\hline 520 & 316.30 & 415.20 & 495.60 \\
\hline 540 & 328.80 & 432 & 544.80 \\
\hline 560 & 320.40 & 446.40 & 529.20 \\
\hline 580 & 337.20 & 462.20 & 634.80 \\
\hline 600 & 358.80 & 480 & 609.60 \\
\hline
\end{tabular}

Table 2 is representing the delay due to spin locking in all the three protocols. It is clearly shown that the delay in Optimal slots are quite less than the other protocols.
Table 3 Delay due to early request finish

\begin{tabular}{|l|l|l|l|}
\hline $\begin{array}{l}\text { No. of } \\
\text { tries }\end{array}$ & $\begin{array}{l}\text { Optimal } \\
\text { slots }\end{array}$ & Slotted & $\begin{array}{l}\text { Un- } \\
\text { slotted }\end{array}$ \\
\hline 300 & 0 & 50 & 70 \\
\hline 320 & 0 & 51 & 75 \\
\hline 340 & 0 & 56 & 96 \\
\hline 360 & 0 & 56 & 87 \\
\hline 380 & 0 & 64 & 72 \\
\hline 400 & 0 & 72 & 93 \\
\hline 420 & 0 & 71 & 106 \\
\hline 440 & 0 & 72 & 108 \\
\hline 460 & 0 & 83 & 116 \\
\hline 480 & 0 & 75 & 115 \\
\hline 500 & 0 & 92 & 136 \\
\hline 520 & 0 & 80 & 123 \\
\hline 540 & 0 & 88 & 106 \\
\hline 560 & 0 & 88 & 136 \\
\hline 580 & 0 & 93 & 172 \\
\hline 600 & 0 & 98 & 158 \\
\hline
\end{tabular}

Table 3 is indicating towards the delay due to early request finish. As mentioned earlier due to better decision making optimal slotted CSMA/CA shows better results as delay due to early finish is 0 .

Fig 5 shows the graphical comparison of throughput of slotted CSMA/CA, un-slotted CSMA/CA and the proposed algorithm. It is clearly shown that the throughput of proposed algorithm is quite better than available protocols as the bar for optimal slot shows the maximum value of throughput for all no. of tries.

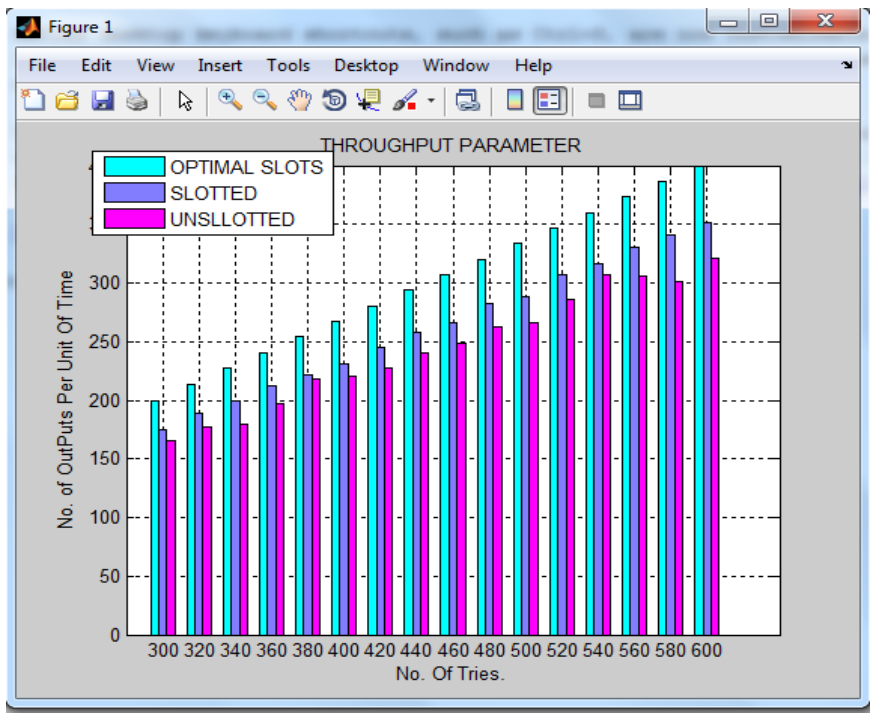

Fig 5 Throughput Analysis

Fig 6 shows the delay due to spin locking in all the three protocols. It is clear from the fig that the delays in Optimal slots are quite less as compared to other protocols as optimal 
slotted protocol's bar shows lowest value of delay due to spinlocking for all no. of tries .

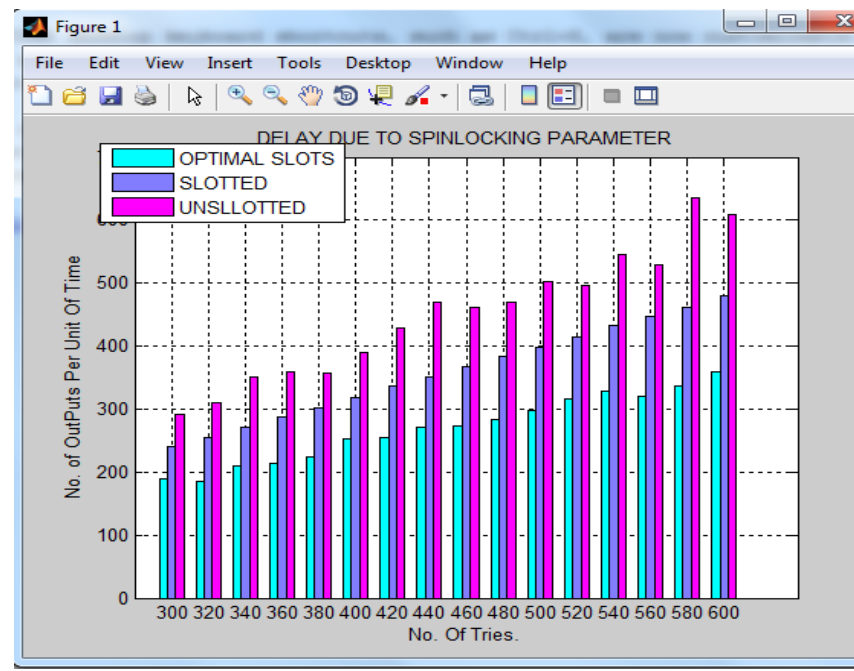

Fig 6 Delay due to spin locking

Fig 7 is showing the delay due to early request finish. As mentioned earlier due to better decision making optimal slotted CSMA/CA shows better results as delay due to early finish is 0 .Therefore the under drawn graph do not show any evidence of delay due to early request finish as it is 0 . So the optimal slotted protocols line never grows with respect to scalability of the network requests.

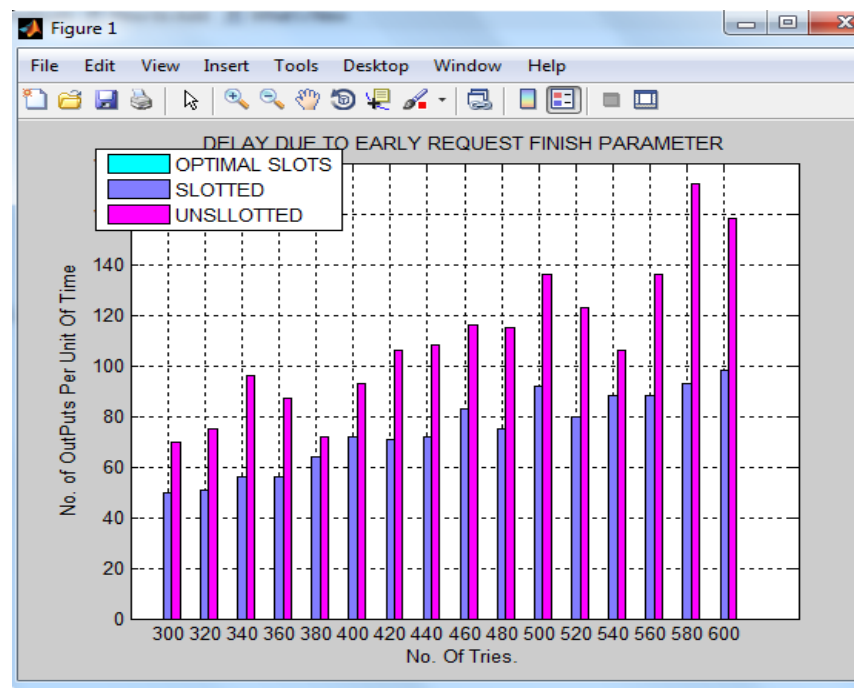

Fig 7 Delay due to early request finish

\section{CONCLUSION}

This paper has proposed a new optimal slotted CSMA/CA which is adaptive to select slot time on run time. So it can reduce the delay time (approx zero) due to early finish of requests. The proposed protocol is implemented in MATLAB and checked on different number of tries. The proposed protocol has ability to remove the early request finish delay time because it selects slots based upon the currently running requests. Comparison among proposed and existing protocols has shown that the proposed protocol provide quite better results than existing protocols. In near future we will implement this protocol in real time environment.

\section{ACKNOWLEDGMENTS}

My sincere thanks to my Co-guides and friends who have contributed towards successful completion of this work.

\section{REFERENCES}

[1] Zhang, Yijin, Pingping $\mathrm{Xu}$, Zaichen Zhang, and Guangguo Bi. 2006"Throughput Analysis of IEEE 802.15. 4 Slotted CSMA/CA Considering Timeout Period and Its Improvement." In Communication systems, 2006. 10th IEEE Singapore International Conference on, pp. 1-5. IEEE, 2006.

[2] Pollin, Sofie, Mustafa Ergen, Sinem Ergen, Bruno Bougard, L. Der Perre, Ingrid Moerman, Ahmad Bahai, Pravin Varaiya, and Francky Catthoor.2008 "Performance analysis of slotted carrier sense IEEE 802.15. 4 medium access layer." Wireless Communications, IEEE Transactions on 7, no. 9, 2008.

[3] Ben othoman jalel.2008. "a multiservice MAC protocol in a multichannel csma/ca for IEEE 802.11 network" In journal of communication and network(volume 10,issue 3)

[4] Wang, Feng, Dou Li, and Yuping Zhao. 2009 "Analysis and compare of slotted and un-slotted CSMA in IEEE 802.15. 4." In Wireless Communications, Networking and Mobile Computing, 2009. WiCom'09. 5th International Conference on, pp. 1-5. IEEE, 2009.

[5] Wen, Hao, Chuang Lin, Zhi-Jia Chen, Hao Yin, Tao He, and Eryk Dutkiewicz .2009."An improved Markov model for IEEE 802.15. 4 slotted CSMA/CA mechanism" Journal of computer science and technology 24, no. 3, 2009.

[6] Wang, Feng, Dou Li, and Yuping Zhao.2010 "An Enhanced Collision-Avoidance MAC Protocol for IEEE 802.15. 4." In Vehicular Technology Conference Fall (VTC 2010-Fall), 2010 IEEE 72nd, pp. 1-5. IEEE, 2010.

[7] Caishi huang and Kowloon. 2010.

"Fairness enhancement for csma/ca wireless network".In systems journal IEEE 2010(Volume:4,issue:4)

[8] Jing, Hui, and Hitoshi Aida. 2011."An analytical approach to optimization of throughput for IEEE 802.15. 4 slotted CSMA/CA networks." In Consumer Communications and Networking Conference (CCNC), 2011 IEEE, pp. 1021-1025.

[9] Alvi, A. N., S. S. Naqvi, S. H. Bouk, N. Javaid, U. Qasim, and Z. A. Khan. 2012. "Evaluation of Slotted CSMA/CA of IEEE 802.15. 4." Seventh International Conference on, pp. 391-396. IEEE, 2012.In Broadband Wireless Computing, Communication and Applications (BWCCA) 